\title{
鄂西南亚高山湿地泥炭藓的生长与分解
}

\author{
刘雪飞 $1^{1,2}$ 吴 林 $^{1,2^{*}}$ 王 涵 1,2 洪 柳 1,2 熊莉军 ${ }^{3}$
}

${ }^{1}$ 生物资源保护与利用湖北省重点实验室, 湖北恩施 $445000 ;{ }^{2}$ 湖北民族大学林学园艺学院, 湖北恩施 $445000 ;{ }^{3}$ 湖北省恩施土家族苗族自治州气象局, 湖北恩施 445000

摘 要 泥炭蘚(Sphagnum)是湿地土壤碳的重要来源, 在土壤碳累积过程中发挥着关键作用, 但有关亚热带湿地泥炭藓生长 与分解的研究鲜有报道。该研究选择鄂西南亚高山泥炭藓湿地为研究区域, 原位开展不同微生境泥炭藓的生长及其调落物的 分解实验, 室内测试调落物的化学成分, 探讨亚热带亚高山湿地泥炭藓的生长与分解规律。结果表明: 泥炭藓在自然状态生 长12个月后, 丘上和丘间两种微生境下泥炭藓的平均高度增长量分别为 2.9 和 $2.7 \mathrm{~cm}$, 对应的净生产量分别为 270.94 和 $370.88 \mathrm{~g} \cdot \mathrm{m}^{-2}$, 生长时间与微生境对泥炭藓的高度增长量及净生产量均有显著影响, 且两者之间存在交互作用, 但是两种微生境下泥炭藓的 生长变化过程不同; 两种微生境下泥炭藓的平均生长速率(2017年7-10月)为 $0.33 \mathrm{~mm} \cdot \mathrm{d}^{-1}$, 其生长速率高于寒温带地区。另外, 分解时间对泥炭藓的分解量有显著影响, 其残留率随时间增加表现为先减少后增加的趋势。12个月后，丘间、丘上和水坑3 种微生境下最终残留率分别为 $100.67 \%$ 、9 $0.54 \%$ 和 $85.63 \%$ 。调落物中碳含量、碳氮比和多酚含量相比初始值均有所下降, 氮 含量则为增加。同时, 微生境对调落物分解的影响取决于分解时间。分解 3 个月时, 微生境之间凋落物的分解量差异显著, 其 他时间段差异不明显。

关键词 亚热带; 泥炭藓; 微生境; 净生产量; 分解

刘雪飞, 吴林, 王涵, 洪柳, 熊莉军 (2020). 鄂西南亚高山湿地泥炭蘚的生长与分解. 植物生态学报, 44, 228-235. DOI: 10.17521/cjpe.2019.0316

\section{Growth and decomposition characteristics of Sphagnum in a subalpine wetland, southwestern Hubei, China}

\author{
LIU Xue-Fei ${ }^{1,2}$, WU Lin ${ }^{1,2^{*}}$, WANG Han ${ }^{1,2}$, HONG Liu $^{1,2}$, and XIONG Li-Jun ${ }^{3}$
}

${ }^{1}$ Hubei Key Laboratory of Biological Resources Protection and Utilization, Enshi, Hubei 445000, China; ${ }^{2}$ College of Forestry and Horticulture, Hubei Minzu University, Enshi, Hubei 445000, China; and ${ }^{3}$ Meteorological Bureau of Enshi Tujia and Miao Autonomous Prefecture, Enshi, Hubei 445000, China

\begin{abstract}
Aims We investigated the growth and decomposition of Sphagnum, a principal plant involved in peat accumulation in wetlands, in subtropical wetlands.

Methods We selected a Sphagnum-dominated wetland in southwestern Hubei Province as the study area. In situ growth and litter decomposition experiments in different microhabitats were carried out, and the chemical composition of litter was measured in laboratory to examine the processes of its growth and decomposition.

Important findings The average height of Sphagnum in hummock and hollow were 2.9 and $2.7 \mathrm{~cm}$, and the net production was 270.94 and $370.88 \mathrm{~g} \cdot \mathrm{m}^{-2}$, respectively, after 12 months. Growth time and microhabitats significantly affected on the height growth and the net production, and there was an interaction between time and microhabitats. However, the growth trend in two microhabitats was significantly different. The average growth rate of Sphagnum under the two microhabitats (July to October 2017) was $0.33 \mathrm{~mm} \cdot \mathrm{d}^{-1}$, which was higher than that in the cold temperate zone. In addition, the decomposition time had an significant effect on the decomposition amount of Sphagnum, and the residual rate decreased first and then increased with time. By the end 12-month period, the final residual rates in hollow, hummock and pool were $100.67 \%, 90.54 \%$ and $85.63 \%$, respectively. The content of carbon $(\mathrm{C})$, carbon nitrogen $(\mathrm{N})$ ratio $(\mathrm{C}: \mathrm{N})$ and polyphenols in the litters decreased, while the nitrogen content increased. During the first 3 months of decomposition, a significant difference of decomposed litter was found between the microhabitats, but no significant difference later on.
\end{abstract}

Key words subtropical; Sphagnum; microhabitats; net production; decomposition

收稿日期Received: 2019-11-19 接受日期Accepted: 2020-01-19

基金项目: 国家自然科学基金(41867042)和湖北省自然科学基金(2017CFB395)。Supported by the National Natural Science Foundation of China (41867042), and the Natural Science Foundation of Hubei Province (2017CFB395).

* 通信作者Corresponding author (wulin20054557@163.com) 
Liu XF, Wu L, Wang H, Hong L, Xiong LJ (2020). Growth and decomposition characteristics of Sphagnum in a subalpine wetland, southwestern Hubei, China. Chinese Journal of Plant Ecology, 44, 228-235. DOI: 10.17521/cjpe.2019.0316

泥炭藓湿地是由适应能力强和耐周期性干旱的 泥炭藓属(Sphagnum)植物以及草本植物沼泽化形成 的一种特殊湿地类型(中国湿地植被编辑委员会, 1999)。由于其净初级生产力 (NPP)大于分解力, 持 续吸收大气中的 $\mathrm{CO}_{2}$, 并积累了大量的泥炭 (Gorham, 1991; Yu, 2012), 因此, 在全球碳循环中发挥 着重要作用。泥炭藓属植物作为泥炭藓湿地的优势 植物，在部分地区其盖度可达 $100 \%$, 其植物残体能 为湿地提供巨大的生物量(Clymo \& Hayward, 1982; Orwin \& Ostle, 2012), 储存在泥炭藓属植物及其残 体中的碳甚至高于每年固定在所有陆地植物体中的 碳量(Clymo \& Hayward, 1982)。泥炭藓属植物是湿 地土壤碳的重要来源, 其固定的碳占据了全球土壤 碳库的30\% (Yu et al., 2010), 在土壤碳累积过程中 发挥着关键作用(Li et al., 2004; Lang et al., 2009)。 因此, 研究泥炭藓的生长及调落物的分解对于揭示 湿地碳循环具有重要意义。

近年来, 国内外学者对泥炭藓属植物的生长及 调落物的分解已开展了大量的研究(Moore, 1989; Rochefort et al., 1990; Gunnarsson, 2005; Breeuwer et al., 2008; Loisel et al., 2012; 李伟等, 2013; Philben et al., 2015; Bengtsson et al., 2016; Mironov \& Kondratev, 2017; Bell et al., 2018; 刘媛媛等, 2018; Newman et al., 2018)。然而这些研究主要集中在高 纬度的寒温带地区, 在低纬度的亚热带地区却鲜有 报道(马进泽, 2018), 有关亚热带地区泥炭藓的生长 及调落物分解的原位研究更是欠缺。事实上, 泥炭 藓属植物的生长及调落物的分解主要受年平均气 温、降水、植被类型、氮沉降等因素的影响(Breeuwer et al., 2008; 李伟等, 2013; Bengtsson et al., 2016; Leroy et al., 2017)。比如Breeuwer等(2008)在西欧的 研究表明, 维管植物的分解速率随气温升高而增加, 而泥炭藓残体的分解速率随气温升高变化不明显, 甚至有减小趋势。相反, 我国学者在长白山的研究 结果表明增温能够促进泥炭藓调落物的分解(李伟 等, 2013)。不同地区的泥炭藓植物生长及调落物分 解的规律并不一致, 这与研究区气候条件、微生境、 植物本身属性等有关(Loisel et al., 2012; 刘媛媛等, 2018)。亚热带地区年平均气温明显高于寒温带地区, 较高的有效积温和充足的降水更有利于泥炭藓的生
长。因此, 我们推测亚热带地区泥炭藓的生长及调 落物的分解将不同于寒温带地区, 甚至亚热带地区 泥炭藓的生长速率高于寒温带地区。

本文选择鄂西南亚高山泥炭藓湿地为研究区域, 原位开展不同微生境下泥炭藓的生长及调落物的分 解实验, 室内测试分析调落物的化学成分, 以探讨 亚热带亚高山地区泥炭藓的生长与分解规律, 旨在 弥补该区域相关研究的不足, 同时为亚热带亚高山 地区泥炭蘚湿地的形成发育规律提供一定的数据 支撑。

\section{1 材料和方法}

\section{1 研究区概况}

研究区位于湖北省恩施市新塘乡太山庙地区 $\left(30.17^{\circ} \mathrm{N}, 109.73^{\circ} \mathrm{E}\right)$, 海拔1 920-1 $970 \mathrm{~m}$, 年平均 气温 $7.2{ }^{\circ} \mathrm{C}$, 且冬季有 $1-2$ 个月的积雪期。年降水量 1550-1 $630 \mathrm{~mm}$, 降水主要集中在夏季, 属于典型 的亚热带湿润山地气候。研究区内有多处呈斑块状 分布的泥炭蘚湿地, 总面积大约 $40 \mathrm{hm}^{2}$ 。湿地内植 被组成丰富多样, 主要由灌木、草本及藓类植物组 成。其中, 灌木群落以杜鹃花科和菩薇科植物为优 势种，草本植物以莎草科和禾本科植物为优势种; 藓类植物主要以泥炭藓(Sphagnum palustre)为优势 种，其盖度可达 $90 \%-100 \%$, 生长高度为 $10-20 \mathrm{~cm}$, 局部区域还发育有高 $20 \mathrm{~cm}$ 左右的藓丘, 湿地中央 分布有常年积水的水坑(图1)。土壤类型主要为酸性 泥炭土，泥炭层厚度为50-110 cm; 土壤表层常年处 于过湿或积水状态，地下水位变化较小，0-50 cm土 壤 具体理化性质分别为: $\mathrm{pH}$ 为 4.16 , 土壤容重为 $0.22 \mathrm{~g} \cdot \mathrm{cm}^{-3}$, 总有机碳为 $249.36 \mathrm{~g} \cdot \mathrm{kg}^{-1}$, 可溶性有机 碳为 $50.05 \mathrm{mg} \cdot \mathrm{L}^{-1}$, 总氮为 $9.02 \mathrm{~g} \cdot \mathrm{kg}^{-1}$ 。

\section{2 研究方法}

\subsection{1 泥炭藓生长实验}

由于常年积水的水坑中未生长泥炭藓, 因此泥 炭藓的生长实验选择在丘间与丘上两种微生境进 行。于2017年7月8日在太山庙泥炭蘚湿地布置泥炭 藓生长监测装置, 在布置时尽量减少对泥炭藓的破 坏, 以保证更加真实地观测泥炭藓在野外条件下的 自然生长规律。泥炭藓的生长测量方法参照Clymo (1970)。即: 将4根长约 $40 \mathrm{~cm}$ 的竹签离顶端 $5 \mathrm{~cm}$ 处刻 
上凹槽，将铁丝牢牢缠绕在凹槽处以防上下滑动, 并使每根竹签之间的间距为 $5 \mathrm{~cm}$ 。将生长监测装置 插入长势均匀的泥炭藓从中, 使泥炭藓顶端位置和 铁丝缠绕位置在同一水平面上，将此位置记为 0 点。 每个微生境中布置 5 个生长观测点, 每 3 个月用直尺 测定一次, 以泥炭藓顶端超过 0 点的长度作为泥炭 藓的生长高度 $(H, \mathrm{~cm})$ 。生长一年后, 记录每个生长 观测点中的泥炭藓株数 $(N)$ 。选取 40 株用收割法收获 地上绿色部分植株, 于室内剪成长度为 $1 、 2 及 3 \mathrm{~cm}$ 等若干片段, 于 $75{ }^{\circ} \mathrm{C}$ 烘干至恒质量, 称量并记录泥 炭藓单位长度的质量 $\left(\mathrm{g} \cdot \mathrm{cm}^{-1}\right)$ 。泥炭藓的 $N P P\left(\mathrm{~g} \cdot \mathrm{m}^{-2}\right)$ 用生长高度与单位长度的质量及单位面积的株数的 乘积进行估算。

\subsection{2 泥炭藓分解实验}

2017年5月初, 于太山庙泥炭藓湿地地中采集 鲜活泥炭藓, 去掉泥炭藓植株下端枯死部分, 统一 保留绿色部分。室内将绿色鲜活泥炭藓于 $75{ }^{\circ} \mathrm{C}$ 下烘 干至恒质量, 然后剪成 $2 \mathrm{~cm}$ 片段。混匀后, 称取 $2 \mathrm{~g}$ 装入大小为 $10 \mathrm{~cm} \times 15 \mathrm{~cm}$ 的双面孔尼龙网袋(粗面 孔径为 18 目, 细面孔径为 80 目)中。于 2017 年7月 8 日 将尼龙袋分别埋入丘上、丘间及水坑 3 种微生境中 (图1), 丘上与丘间生境所埋深度为距地表 $5 \mathrm{~cm}$ 左右 位置, 水坑生境将尼龙袋全部浸泡在水深 $5 \mathrm{~cm}$ 处。 埋尼龙网袋时, 用手将袋中的泥炭藓压平整, 尽量 使植物体在袋中均匀分布, 同时细面孔径朝下, 粗 面孔径朝上, 做好标记后用尼龙线系于竹笔上并固 定。每3个月取一次分解袋, 取样时间依次为 2017 年 10月8日、2018年1月7日、2018年4月7日、2018年7 月 8 日。每次每个微生境取回3袋, 共计 36 袋 $(3$ 种微 生境 $\times 4$ 次取样 $\times 3$ 个重复)。
将取回的尼龙网袋于室内, 清除表面上的泥土 和植物根系后, 置于 100 目篮(孔径 $0.15 \mathrm{~mm}$ )中, 用 蒸馏水缓慢冲洗, 冲洗干净后于 $75{ }^{\circ} \mathrm{C}$ 烘干至恒质 量, 称量记录凋落物的残留量。将残留的调落物用 粉碎机粉碎, 过50目篮后, 用元素分析仪(Elementar Vario EL III, Elementar, Langenselbold, Germany)测 定残留物中的总碳 $(\mathrm{C})$ 和总氮 $(\mathrm{N})$ 含量, 用FolinCiocalteu法测多酚含量(Singleton et al., 1999)。

调落物的分解程度通过质量残留率 $(E)$ 表示:

$$
E=M_{t} / M_{0} \times 100 \%
$$

式中, $M_{0}$ 为调落物初始质量 $(\mathrm{g}), M_{t}$ 为调落物分解后 的剩余质量 $(\mathrm{g}) 。 E$ 值越大, 分解越慢; $E$ 值越小, 分解 越快。

\subsection{3 数据分析}

泥炭藓生长在不同时间与不同微生境之间的差 异性使用重复测量方差分析和最小显著差异法 $(L S D)$ 进行显著性检验 $(p=0.05)$, 并对变量进行球 形检验，发现球形检验的 $p$ 值小于 0.05 , 表明泥炭藓 的生长在不同时间之间存在相关性，且生长时间与 微生境之间存在交互作用，需要使用多变量方差分 析。而球形检验结果表明调落物分解在不同时间不 存在相关性 $(p>0.05)$, 分解时间与微生境之间不存 在交互作用。因此, 泥炭藓调落物的分解在不同时 间与不同微生境之间的差异性使用单因素方差分析 和 $L S D$ 进行显著性检验 $(p=0.05)$, 所有数据在分析 之前进行Shapiro-Wilk正态性检验，并对不符合正 态分布的数据进行基于 $\lg (x+k)$ 或 $1 / x$ 的数据转换。 数据分析使用SPSS 24.0 完成, 并用Origin 2018 绘图。


图1 鄂西南亚高山泥炭藓湿地中的3种微生境(从左至右依次为: 丘上、丘间、水坑)。

Fig. 1 Three types of microhabitats in Sphagnum-dominated subalpine wetland in southwestern Hubei (From left to right: hummock, hollow, and pool).

www.plant-ecology.com 


\section{2 结果和分析}

\section{1 泥炭藓的生长规律}

太山庙泥炭藓湿地中, 丘上和丘间两种微生境 下泥炭蘚生长 12 个月后的平均高度增长量分别为 2.9 和 $2.7 \mathrm{~cm}$, 对应的净生产量分别为 270.94 和 $370.88 \mathrm{~g} \cdot \mathrm{m}^{-2}$ 。生长时间与微生境对泥炭藓的高度增 长量及净生产量均有显著影响 $(p<0.05)$, 且两者之 间存在交互作用 $(p<0.05)$ 。除生长 12 个月外, 两种 微生境下泥炭藓的高度增长量及净生产量在其他时 间存在显著差异 $(p<0.05)$ 。但是, 两种微生境下泥 炭藓的生长规律表现出不同的趋势。丘上生境下泥 炭蘚生长表现为持续性增长, 丘间生境下则表现为 先增加后减少。在生长的前 9 个月之内, 丘间生境的 生长速率明显高于丘上生境 $(p<0.05)$, 丘上生境的 生长速率为丘间生境的 $26.90 \%$ 。而在生长 12 个月后, 两种生境的生长速率基本一致且无明显差异(图2)。

\section{2 泥炭藓的分解规律}

总体上，分解时间对泥炭藓的分解量有显著影 响 $(p<0.05)$, 即分解 12 个月后, 泥炭藓最终残留率 平均为 $92.28 \%$ 。3种生境下, 泥炭藓最终残留率并不 随时间增加简单呈现出递减趋势，而是表现为先减 少后增加。其中丘间生境下的最终残留率达到 $100.67 \%$ ，较初始值的 $100 \%$ 有所增加，而丘上生境 与水坑生境均有所下降，分别为 $90.54 \%$ 和 $85.64 \%$

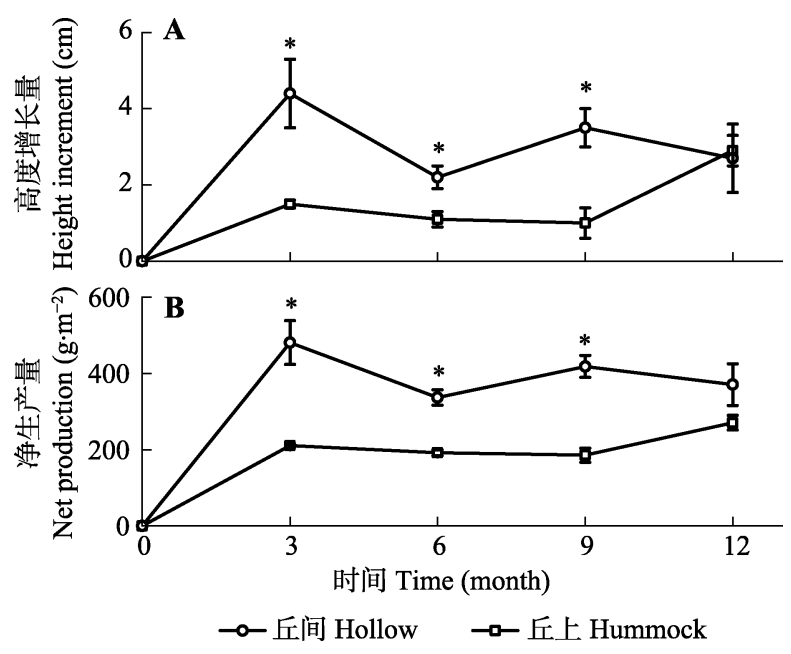

图2 鄂西南亚高山泥炭藓湿地丘间与丘上两种微生境下泥 炭藓高度增长量 $(\mathbf{A})$ 及净生产量 $(\mathbf{B})$ 变化(平均值土标准误差, $n=$ $3)$ 。表示在同一时间不同微生境下有显著性差异 $(p<0.05)$ 。 Fig. 2 Increment of height growth (A) and net production (B) of Sphagnum in hollow and hummock of Sphagnum-dominated subalpine wetlands in southwestern Hubei (mean $\pm S E, n=3$ ). * refers a significant difference between the two microhabitats at the same time $(p<0.05)$.
(图3)。此外，泥炭藓调落物的 C、N含量、C:N和多 酚含量表现为波动性的变化, $\mathrm{C}$ 含量、 $\mathrm{C}: \mathrm{N}$ 和多酚含 量的平均值较初始值分别下降 $10.69 \%$ 、17.78\% 和 $29.1 \%, \mathrm{~N}$ 含量的平均值较初始值增长 $9.57 \%$ (图4)。

另外，微生境对泥炭藓调落物的分解有一定影 响，但是不同时间段影响程度不同。分解 3 个月时, 3 种微生境下调落物残留量存在显著差异 $(p<0.05)$, 其他时间不存在显著差异。整体上，水坑生境下泥 炭藓调落物的分解速率最快，丘间生境下则分解最 慢(图3)。泥炭藓最终的残留率、C含量、N含量、 $\mathrm{C}: \mathrm{N}$ 及多酚含量等均不存在显著性差异，但在某一 时间段上存在显著性差异 $(p<0.05$ )(图3，图4)。

\section{3 讨论}

\section{1 泥炭藓的生长及影响因素}

我们推测亚热带地区泥炭藓的生长及调落物的 分解将不同于寒温带地区, 甚至亚热带地区泥炭藓 的生长速率高于寒温带地区。本研究中两种微生境 下泥炭藓在生长季节(2017年7-10月)的平均高度生 长速率及净生产力明显高于其他高纬度寒温带地区 (表1), 这一结果证实了我们的假设。事实上，我们 进一步分析泥炭藓属植物生长速率及净生产力与纬



图3 鄂西南亚高山泥炭藓湿地3种微生境下泥炭藓调落物 残留率变化规律(平均值土标准误差, $n=3$ )。*表示在同一时 间不同微生境下有显著性差异 $(p<0.05)$, 不同小写字母表 示在同一微生境不同时间有显著性差异 $(p<0.05)$ 。

Fig. 3 Changes in litters residual rate of Sphagnum under three microhabitats of Sphagnum-dominated subalpine wetlands in southwestern Hubei (mean $\pm S E, n=3$ ). * refers a significant difference among the three microhabitats at the same time $(p<$ 0.05 ); different lowercase letters indicate significant differences at different time periods in the same microhabitat $(p<0.05)$. 

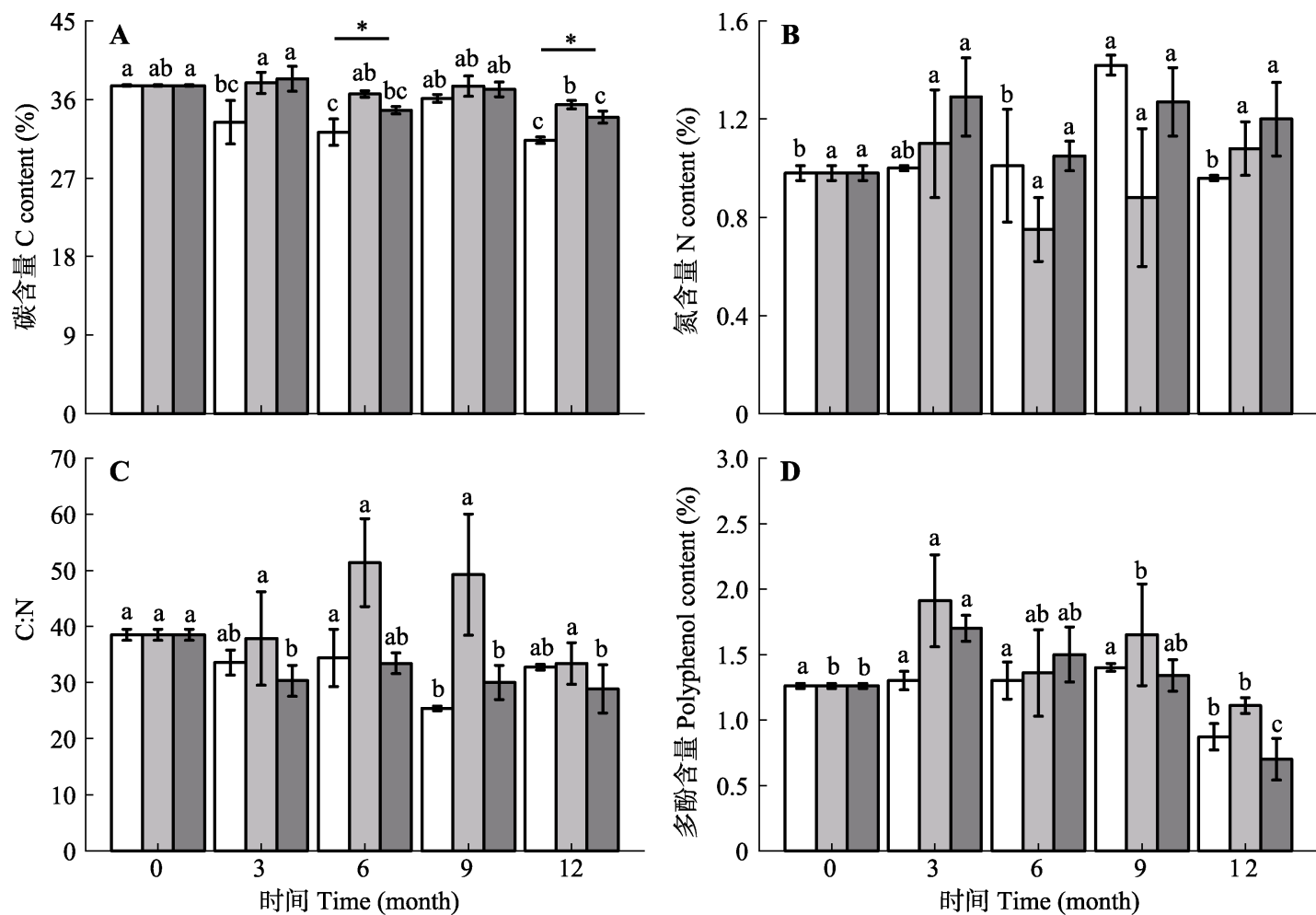

丘间 Hollow

丘上 Hummock

水坑 Pool

图4 鄂西南亚高山泥炭藓湿地3种微生境下泥炭藓调落物 $\mathrm{C}(\mathbf{A}) 、 \mathrm{~N}(\mathbf{B}) 、 \mathrm{C}: \mathrm{N}(\mathbf{C})$ 及多酚含量 $(\mathbf{D})$ 变化规律 $($ 平均值 \pm 标准误差, $n=$ $3)$ 。*表示在同一时间不同微生境下有显著性差异 $(p<0.05)$, 不同小写字母表示在同一微生境不同时间有显著性差异 $(p<$ $0.05)$ 。

Fig. 4 Contents of C (A), N (B), C:N (C) and polyphenols (D) in the litters of Sphagnum of the three microhabitats of Sphagnumdominated subalpine wetlands in southwestern Hubei (mean $\pm S E, n=3)$ * refers a significant difference among the three microhabitats at the same time $(p<0.05)$; different lowercase letters indicate significant differences at different time periods in the same microhabitat $(p<0.05)$.

表1 不同纬度地区泥炭藓属植物的生长速率及净生产力

Table 1 Growth rate and net primary productivity of Sphagnum spp. in areas of different latitude

\begin{tabular}{|c|c|c|c|c|c|c|c|}
\hline $\begin{array}{l}\text { 纬度 } \\
\text { Latitude } \\
\left({ }^{\circ}\right)\end{array}$ & $\begin{array}{c}\text { 经度 } \\
\text { Longitude }\left(^{\circ}\right)\end{array}$ & $\begin{array}{c}\text { 年平均气温 } \\
\text { Annual mean } \\
\text { temperature }\left({ }^{\circ} \mathrm{C}\right)\end{array}$ & $\begin{array}{c}\text { 年降水量 } \\
\text { Precipitation }(\mathrm{mm})\end{array}$ & $\begin{array}{l}\text { 物种 } \\
\text { Species }\end{array}$ & $\begin{array}{c}\text { 生长速率 } \\
\text { Growth rate } \\
\left(\mathrm{mm} \cdot \mathrm{d}^{-1}\right)\end{array}$ & $\begin{array}{c}\text { 净生产力 } \\
\text { Net primary produc- } \\
\text { tivity }\left(\mathrm{g} \cdot \mathrm{m}^{-2} \cdot \mathrm{d}^{-1}\right)\end{array}$ & $\begin{array}{l}\text { 参考文献 } \\
\text { Reference }\end{array}$ \\
\hline 68.35 & 18.82 & 0.5 & 320 & S. fus & 0.04 & 1.51 & Dorrepaal et al., 2003 \\
\hline 60.52 & 17.92 & 3.1 & 544 & S. fus & 0.10 & 0.64 & Bengtsson et al., 2016 \\
\hline 59.90 & 15.83 & 1.4 & 515 & S. fus, S. mag & 0.13 & 1.12 & Bengtsson et al., 2016 \\
\hline 57.15 & -111.98 & 2.1 & 387 & S. fus & 0.09 & 1.68 & Wieder et al., 2010 \\
\hline 56.63 & -110.20 & 1.1 & 420 & S. fus & 0.09 & 1.72 & Wieder et al., 2010 \\
\hline 55.68 & -111.83 & 2.1 & 421 & S. fus & 0.10 & 1.96 & Wieder et al., 2010 \\
\hline 54.68 & -113.47 & 1.7 & 500 & S. fus & 0.03 & 1.15 & Thormann \& Bayley, 1997 \\
\hline 49.67 & -93.72 & 2.6 & 714 & S. mag, S. fus, S. ang & 0.12 & 0.86 & Rochefort et al., 1990 \\
\hline 46.32 & 11.67 & 5.0 & 1100 & S. cap, S. mag, S. fal & 0.26 & 1.70 & Gerdol, 1995 \\
\hline 39.12 & -79.58 & 7.9 & 1330 & S. mag, S. rec & 0.42 & 3.44 & Wieder \& Lang, 1983 \\
\hline 30.17 & 109.73 & 7.2 & 1590 & S. pal & 0.33 & 3.84 & 本研究 This study \\
\hline
\end{tabular}

S. ang, 小叶泥炭藓; S. cap, 尖叶泥炭鲜; S. fal, 假泥炭藓; S. fus, 锈色泥炭藓; S. mag, 中位泥炭藓; S. pal, 泥炭藓; S. rec, 喙叶泥炭藓。泥炭藓属植物学 名及拉丁名参照王利松等(2018)。泥炭藓属植物的生长数据均为采用Clymo (1970)方法进行野外原位测定所得, 测定时间均为生长季节, 且生长速率及 净生产力统一转换为 $\mathrm{mm} \cdot \mathrm{d}^{-1}$ 和 $\mathrm{g} \cdot \mathrm{m}^{-2} \cdot \mathrm{d}^{-1}$ 。

S. ang, S. angustifolium; S. cap, S. capillifolium; S. fal, S. fallax; S. fus, S. fuscum; S. mag, S. magellanicum; S. pal, S. palustre; S. rec, S. recurvum. Scientific and Latin names of Sphagnum spp. refer to Wang et al. (2018). The growth data of Sphagnum spp. in the table were all obtained by field measurement using Clymo (1970) method. Measuring time covers whole growing season; the growth rate and net primary productivity were converted to $\mathrm{mm} \cdot \mathrm{d}^{-1}$ and $\mathrm{g} \cdot \mathrm{m}^{-2} \cdot \mathrm{d}^{-1}$. 
度关系时, 发现生长速率及净生产力与纬度呈显著 负相关关系(表1; 图5), 即随着纬度的降低, 泥炭藓 属植物的生长速率和净生产力会增加, 这与相关学 者在寒温带地区的研究结果(Wieder \& Lang, 1983) 一致。重要的是, 本研究将这一纬度变化规律从寒 温带外推到寒温带至亚热带。

本研究区泥炭藓高度生长速率及净生产力高于 寒温带地区, 可能与研究区年平均气温及降水量较 高有关。有研究表明, 泥炭藓的高度生长受气温及 降水共同调控(Kosykh et al., 2017)。Kosykh等(2017) 在西伯利亚西部的研究表明泥炭藓的高度生长对气 温有依赖性, 但在不同地区气温与降水的影响程度 不同。在北美和欧洲不同气候条件下，气温是影响 锈色泥炭藓(S. fuscum)和中位泥炭藓(S. magellanicum)生长的最重要因素, 而湿度的作用不显著 (Loisel et al., 2012)。然而Asada等(2003)在加拿大的 研究表明, 在适于泥炭藓生长的气温范围内, 降水 量对生长的影响更大, 当气温超过泥炭藓生长的最 适温度时, 降水量对生长无显著作用。另外, 泥炭藓 属植物生长与物种及生境有关。本研究区泥炭藓物 种为泥炭藓, 不同于寒温带地区广泛分布的锈色泥 炭蘚和中位泥炭蘚(表1)。另外, 本研究中泥炭藓在 丘间生境下的生长速率及净生产量显著高于丘上生 境, 这与众多的研究结果一致, 这可能与不同微生 境下水分差异有关(Moore, 1989; Gerdol, 1995; Asada et al., 2003)。

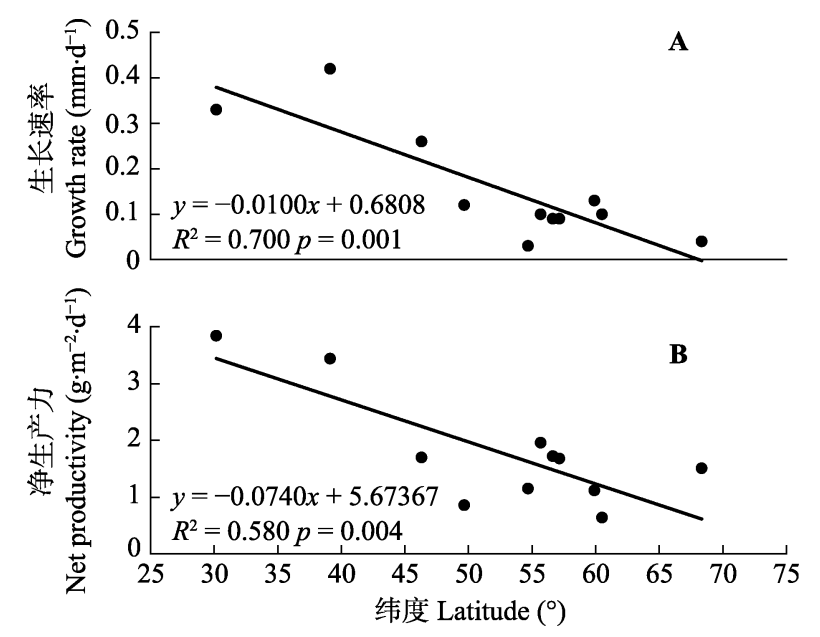

图5 泥炭藓属植物生长速率 $(\mathbf{A})$ 及净生产力 $(\mathbf{B})$ 与纬度之间 的关系。

Fig. 5 Rrelationships between growth rate (A) and net primary productivity (B) of Sphagnum spp. and latitude.
本研究中泥炭藓的生长变化规律随时间的不同 表现出季节性变化规律, 这与以往的研究结果 (Furness \& Grime, 1982; Moore, 1989; Genet et al., 2013; Mironov \& Kondratev, 2017)相同, 且微生境 与生长时间对泥炭藓的生长有一定的交互作用。有 研究表明, 泥炭藓的季节性生长模式与季节性温差 有显著相关性(Mironov \& Kondratev, 2017)。另外, 本研究中, 泥炭藓在生长 6 个月(即2018年1月)时, 丘上与丘间生境泥炭藓的高度下降率分别为 $27.10 \%$ 和 $51.13 \%$, 这可能与该区域冬季降雪有关。 有研究发现, 冬季积雪会显著降低泥炭藓的高度 (Waddington et al., 2003; Yazaki \& Yabe, 2012), 甚 至导致生物量减少。

\section{2 泥炭藓的分解规律及其影响因素}

泥炭藓的分解相比生长更为复杂, 其分解过程 不仅受地表植被类型、生境、气候条件及氮沉降等 环境因素的影响(Gerdol et al., 2007; Breeuwer et al., 2008; 李伟等，2013)，同时也受不同物种间本身属 性的差异(李伟等, 2013; Bengtsson et al., 2018; 刘 媛媛等, 2018)和土壤养分含量的差异(Bragazza et al., 2007)所影响。本研究 3 种微生境下泥炭藓调落物 野外分解 12 个月后最终残留率平均为 $92.28 \%$, 略高 于刘媛媛等(2018)在长白山哈泥泥炭地的研究结果 (90\%)。同样是泥炭藓湿地, 李伟等(2013)在长白山 的研究发现泥炭藓调落物经历 14 个月的分解后最终 残留率为 $80.8 \%$, 显著低于本研究结果。这可能与不 同调落物本身的化学属性及研究区的环境有关。研 究表明, 调落物 C:N 越高, 分解越快(Limpens \& Berendse, 2003)。与李伟等(2013)及刘媛媛等(2018) 在长白山的研究相比, 本研究中泥炭藓初始 $\mathrm{C}: \mathrm{N}$ 较 低, 而分解却更慢, 这可能与本研究区调落物本身 多酚含量更高、土壤pH较低及土壤碳氮含量更低有 一定关系。研究表明, 较高的多酚含量(Jassey et al., 2011)、较低的pH (Tahvanainen \& Haraguchi, 2013) 及较低的土壤养分含量(Bragazza et al., 2007)会抑 制调落物的分解。另外, 温度也是影响调落物分解 的一个重要因素。以往的研究表明, 较高的温度会 促进调落物的分解(Zhang et al., 2017), 也有研究认 为较高的温度对调落物的分解无显著影响(Breeuwer et al., 2008)。本研究区温度显著高于寒温带地 区, 然而分解率却低于寒温带地区, 可能与研究区 其他环境因子对调落物分解的抑制作用较强有关, 
比如研究区土壤酸性高于寒温带地区，调落物多酚 含量也更高。

本研究发现分解时间对泥炭藓的分解量有显著 影响 $(p<0.05)$, 但其残留率随时间增加表现为先减 少后增加。也就是其残留量并不是持续减少的, 甚 至在某个时间段有短暂的增加现象。马进泽(2018) 在大九湖泥炭地也发现泥炭藓调落物分解过程中有 增加情况，甚至分解3年后，最终残留量较初始值有 所增加, 这可能与泥炭藓超强的吸水能力有关。本 研究区与大九湖泥炭地同属鄂西地区, 泥炭藓湿地 环境比较相似, 泥炭层中离子含量较高。因而, 泥炭 藓分解到一定程度后, 其残体有可能吸附了周围环 境中的一定量的化学物质。有研究表明, 泥炭藓能 够从周围环境中吸附大量的阳离子(Ho \& Mckay, 2000)。当然, 这其中的具体原因还有待进一步研究。

另外, 微生境对调落物分解也有一定影响, 但 影响程度取决于分解时间。本研究发现, 分解 3 个月 时, 微生境之间调落物的分解量差异显著, 其他时 间段差异不明显。以往的研究表明, 狭叶泥炭藓和 假泥炭藓(S. fallax)等丘间物种的分解速率高于锈色 泥炭藓和中位泥炭举等丘上物种(Johnson \& Damman, 1993; Limpens \& Berendse, 2003)。然而, 这些 研究在比较生境对分解速率的影响时, 选择的是泥 炭藓属的不同物种, 未比较同一物种在不同生境下 的差异, 这忽略了不同物种本身化学属性上的差异 所带来的影响。而本研究为同一物种在不同生境下 的分解, 最终结果表明微生境对泥炭藓调落物的分 解无显著影响。这可能与本研究中两种微生境环境 差异不大有关。

致谢 感谢湖北民族大学余夏君、吴浩、张宏同学 在野外样品采集和室内样品分析工作中给予的帮助。

\section{参考文献}

Asada T, Warner BG, Banner A (2003). Growth of mosses in relation to climate factors in a hypermaritime coastal peatland in British Columbia, Canada. The Bryologist, 106, 516-527.

Bell MC, Ritson JP, Verhoef A, Brazier RE, Templeton MR, Graham NJD, Freeman C, Clark JM (2018). Sensitivity of peatland litter decomposition to changes in temperature and rainfall. Geoderma, 331, 29-37.

Bengtsson F, Granath G, Rydin H (2016). Photosynthesis, growth, and decay traits in Sphagnum-A multispecies comparison. Ecology and Evolution, 6, 3325-3341.
Bengtsson F, Rydin H, Hájek T (2018). Biochemical determinants of litter quality in 15 species of Sphagnum. Plant and Soil, 425, 161-176.

Bragazza L, Siffi C, Iacumin P, Gerdol R (2007). Mass loss and nutrient release during litter decay in peatland: the role of microbial adaptability to litter chemistry. Soil Biology \& Biochemistry, 39, 257-267.

Breeuwer A, Heijmans M, Robroek BJM, Limpens J, Berendse $F$ (2008). The effect of increased temperature and nitrogen deposition on decomposition in bogs. Oikos, 117, 1258-1268.

Clymo RS (1970). The growth of Sphagnum: methods of measurement. Journal of Ecology, 58, 13-49.

Clymo RS, Hayward PM (1982). The ecology of Sphagnum// Smith AJE. Bryophyte Ecology. Chapman and Hall, London.

Dorrepaal E, Aerts R, Cornelissen JHC, Callaghan TV, van Logtestijn RSP (2003). Summer warming and increased winter snow cover affect Sphagnum fuscum growth, structure and production in a sub-arctic bog. Global Change Biology, 10, 93-104.

Editorial Board of Wetland Vegetation in China (1999). Wetland Vegetation in China. Science Press, Beijing. [中国湿 地植被编辑委员会 (1999). 中国湿地植被. 科学出版 社, 北京.]

Furness SB, Grime JP (1982). Growth rate and temperature responses in bryophytes: I. An investigation of Brachythecium rutabulum. Journal of Ecology, 70, 513-523.

Genet H, Oberbauer SF, Colby SJ, Staudhammer CL, Starr G (2013). Growth responses of Sphagnum hollows to a growing season lengthening manipulation in Alaskan Arctic tundra. Polar Biology, 36, 41-50.

Gerdol R (1995). The growth dynamics of Sphagnum based on field measurements in a temperate bog and on laboratory cultures. Journal of Ecology, 83, 431-437.

Gerdol R, Petraglia A, Bragazza L, Iacumin P, Brancaleoni L (2007). Nitrogen deposition interacts with climate in affecting production and decomposition rates in Sphagnum mosses. Global Change Biology, 13, 1810-1821.

Gorham E (1991). Northern peatlands: role in the carbon cycle and probable responses to climatic warming. Ecological Applications, 1, 182-195.

Gunnarsson U (2005). Global patterns of Sphagnum productivity. Journal of Bryology, 27, 269-279.

Ho YS, Mckay G (2000). The kinetics of sorption of divalent metal ions onto Sphagnum moss peat. Water Research, 34, 735-742.

Jassey VEJ, Chiapusio G, Gilbert D, Buttler A, Toussaint ML, Binet P (2011). Experimental climate effect on seasonal variability of polyphenol/phenoloxidase interplay along a narrow fen-bog ecological gradient in Sphagnum fallax. Global Change Biology, 17, 2945-2957.

Johnson LC, Damman AWH (1993). Decay and its regulation

www.plant-ecology.com 
in Sphagnum peatlands. Advances in Bryology, 5, 249-296.

Kosykh NP, Koronatova NG, Granath G (2017). Effect of temperature and precipitation on linear increment of Sphagnum fuscum and S. magellanicum in Western Siberia. Russian Journal of Ecology, 2017, 48, 203-211.

Lang SI, Cornelissen JHC, Klahn T, van Logtestijn RSP, Broekman R, Schweikert W, Aerts R (2009). An experimental comparison of chemical traits and litter decomposition rates in a diverse range of subarctic bryophyte, lichen and vascular plant species. Journal of Ecology, 97, 886-900.

Leroy F, Gogo S, Guimbaud C, Bernard-Jannin L, Hu Z, LaggounDéfarge F (2017). Vegetation composition controls temperature sensitivity of $\mathrm{CO}_{2}$ and $\mathrm{CH}_{4}$ emissions and DOC concentration in peatlands. Soil Biology \& Biochemistry, 107, 164-167.

Li H, Parent LE, Karam A, Tremblay C (2004). Potential of Sphagnum peat for improving soil organic matter, water holding capacity, bulk density and potato yield in a sandy soil. Plant and Soil, 265, 355-365.

Li W, Bu ZJ, Zhang BJ, Long C, Tang RJ, Cui QW (2013). Decomposition of Sphagnum litter in 4 peatlands of the Changbai Mountains along an altitudinal gradient. Journal of Mountain Science, 31, 442-447. [李伟, 卜兆君, 张兵 将, 龙川, 唐瑞江, 崔钱王 (2013). 长白山不同海拔泥 炭地泥炭蘚残体的分解. 山地学报, 31, 442-447.]

Limpens J, Berendse F (2003). How litter quality affects mass loss and $\mathrm{N}$ loss from decomposing Sphagnum. Oikos, 103, 537-547.

Liu YY, Ma JZ, Bu ZJ, Wang SZ, Zhang XB, Zhang TY, Liu SS, Fu B, Kang Y (2018). Effect of geographical sources and biochemical traits on plant litter decomposition in a peatland. Chinese Journal of Plant Ecology, 42, 713-722. [刘媛媛, 马进泽, 卜兆君, 王升忠, 张雪冰, 张婷玉, 刘莎莎, 付彪, 康媛 (2018). 地理来源与生物化学属性 对泥炭地植物残体分解的影响. 植物生态学报, 42 , 713-722.]

Loisel J, Gallego-Sala AV, Yu Z (2012). Global-scale pattern of peatland Sphagnum growth driven by photosynthetically active radiation and growing season length. Biogeosciences, 9, 2737-2746.

Ma JZ (2018). The Simulation Study on Effects of Climate Warming on Plant Litter Decomposition in Peatlands Basing on Three Experimental Modes. Master degree dissertation, Northeast Normal University, Changchun. [马进泽 (2018). 基于三种实验方式的气候变暖对泥炭地植物调落物分 解影响的模拟研究. 硕士学位论文, 东北师范大学, 长 春.]

Mironov VL, Kondratev AY (2017). Peat moss Sphagnum riparium follows a circatrigintan growth rhythm in situ: a case report. Chronobiology International, 34, 981-984.

Moore TR (1989). Growth and net production of Sphagnum at five fen sites, subarctic eastern Canada. Canadian Journal of Botany, 67, 1203-1207.

Newman TR, Wright N, Wright B, Sjögersten S (2018). Inter- acting effects of elevated atmospheric $\mathrm{CO}_{2}$ and hydrology on the growth and carbon sequestration of Sphagnum moss. Wetlands Ecology and Management, 26, 763-774.

Orwin KH, Ostle NJ (2012). Moss species effects on peatland carbon cycling after fire. Functional Ecology, 26, 829-836.

Philben M, Holmquist J, MacDonald G, Duan DD, Kaiser, K, Benner R (2015). Temperature, oxygen, and vegetation controls on decomposition in a James Bay peatland. Global Biogeochemical Cycles, 29, 729-743.

Rochefort L, Vitt DH, Bayley SE (1990). Growth, production, and decomposition dynamics of Sphagnum under natural and experimentally acidified conditions. Ecology, 71, 1986-2000.

Singleton VL, Orthofer R, Lamuela-Raventós RM (1999). Analysis of total phenols and other oxidation substrates and antioxidants by means of folin-ciocalteu reagent. Methods in Enzymology, 299, 152-178.

Tahvanainen T, Haraguchi A (2013). Effect of pH on phenol oxidase activity on decaying Sphagnum mosses. European Journal of Soil Biology, 54, 41-47.

Thormann MN, Bayley SE (1997). Aboveground net primary production along a bog-fen-marsh gradient in southern boreal Alberta, Canada. Écoscience, 4, 374-384.

Waddington JM, Rochefort L, Campeau S (2003). Sphagnum production and decomposition in a restored cutover peatland. Wetlands Ecology \& Management, 11, 85-95.

Wang LS, Jia Y, Zhang XC, Qin HN (2018). Species Catalogue of China. Volume 1 Plants A Synoptic Checklist (I). Science Press, Beijing. [王立松, 贾谕, 张宪春, 覃海宁 (2018). 中 国生物名录第一卷 植物总名录(上册). 科学出版社, 北 京.]

Wieder RK, Lang GE (1983). Net primary production of the dominant bryophytes in a Sphagnum-dominated wetland in West Virginia. The Bryologist, 86, 280-286.

Wieder RK, Vitt DH, Burke-Scoll M, Scott KD, House M, Vile MA (2010). Nitrogen and sulphur deposition and the growth of Sphagnum fuscum in bogs of the Athabasca Oil Sands Region, Alberta. Journal of Limnology, 69, 161-170.

Yazaki T, Yabe K (2012). Effects of snow-load and shading by vascular plants on the vertical growth of hummocks formed by Sphagnum papillosum in a mire of northern Japan. Plant Ecology, 213, 1055-1067.

Yu ZC (2012). Northern peatland carbon stocks and dynamics: a review. Biogeosciences, 9, 4071-4085.

Yu ZC, Loisel J, Brosseau DP, Beilman DW, Hunt SJ (2010). Global peatland dynamics since the last glacial maximum. Geophysical Research Letters, 37, L13402. DOI: 10.1029/ $2010 \mathrm{~g} 1043584$.

Zhang XH, Sun XX, Mao R (2017). Effects of litter evenness, nitrogen enrichment and temperature on short-term litter decomposition in freshwater marshes of Northeast China. Wetlands, 37, 145-152.

特邀编委: 刘国方 责任编辑: 李 敏 